\title{
DETERMINAÇÃO DO COEFICIENTE DE TRANSFERÊNCIA DE MASSA DO CLORETO DE SÓDIO EM ÁGUA EM SISTEMAS AGITADOS
}

\author{
S.C. Magalhães ${ }^{1}$ \\ L.A. Calçada ${ }^{1}$ \\ C.M. Scheid ${ }^{1}$ \\ J. C. Pinto ${ }^{2}$
}

Resumo: Experimentos de dissolução de cloreto de sódio em água foram conduzidos em duas unidades experimentais como objetivo de avaliar os efeitos da temperatura e grau de agitação na cinética de dissolução. Foram propostos modelos para correlacionar a cinética de dissolução com as condições operacionais utilizadas em ambos os sistemas. Os dados experimentais e os dados gerados pelos modelos apresentaram concordância.

Palavras-chave: perfuração, pré-sal, convecção.

Abstract: Experiments of sodium chloride dissolution were done in two experimental units. The ultimate goal was to evaluate the effects of temperature and degree of turbulence in the dissolution kinetics. Models were proposed to correlate the kinetics with the operational conditions of each experimental system. The experimental data and the ones originated by the proposed models showed agreement.

Keywords: drilling, salt layer, convection.

\footnotetext{
${ }^{1}$ Universidade Federal Rural do Rio de Janeiro, Instituto de Tecnologia, Departamento de Engenharia Química. E-mail: sergiomagalhaes@sergiomagalhaes.eng.br

${ }^{2}$ Instituto Alberto Luiz Coimbra de Pós Graduação e Pesquisa de Engenharia - COPPE-UFRJ.
} 


\section{Introdução}

A indústria petrolífera vem enfrentando diversos desafios técnicos para perfurar poços de petróleo em águas profundas. Durante a perfuração, no cenário do pré-sal, os cascalhos de sal gerados pela broca tendem a se dissolver no fluido de perfuração, o que deve ser controlado a fim de se manter a integridade do poço e a segurança operacional.(Shaughnessy et al., 2007).

Os modelos encontrados na literatura para predição da cinética de dissolução são baseados no balanço de massa entre a fase sólida do sal (retículo cristalino) e a fase líquida do sal (sal dissolvido). Nestes modelos a cinética é representada como uma variável explícita e, dependendo da forma como se coloca o balanço de massa, esta variável pode ser o coeficiente de transferência de massa, o fluxo de liberação de massa ou a taxa de dissolução do sal (Bird et al, 2002).

Para a obtenção da cinética são necessários dados experimentais do processo dissolutivo, o método do disco rotativo é o mais empregado para este fim (Alkattan et. al. 1997).Este método consiste em girar uma única amostra de sal, de geometria definida, em um fluido estacionário. No entanto, na indústria petrolífera, este processo é caracterizado pela dissolução de um conjunto de partículas em um fluido em movimento. Baseado nesta diferença, este trabalho propôs a avaliação da cinética de dissolução em um sistema experimental que se assemelha mais ao processo industrial.

\section{Revisão Bibliográfica}

\subsection{Cinética de dissolução}

Aksel`rud et al. (1992)propuseram um sistema de dissolução em fluxo fechado, por meio de uma unidade experimental montada em bancada. Os autores determinaram a cinética de dissolução de diversos sais em água destilada por meio da equação,

$$
-\frac{\mathrm{dm}_{\text {salt }}}{\mathrm{dt}}=\mathrm{kA}\left(\mathrm{C}^{*}-\mathrm{C}\right),
$$

onde $\mathrm{m}_{\text {salt }}(\mathrm{kg})$ é a massa que deixa o cristal (fase sólida), $\mathrm{t}$ (s) é o tempo, $\mathrm{k}$ $(\mathrm{m} / \mathrm{s})$ é o coeficiente de transferência de massa, A $\left(\mathrm{m}^{2}\right)$ é a área total de transferência de massa, $C^{*}\left(\mathrm{~kg} / \mathrm{m}^{3}\right)$ é a concentração no ponto de saturação $\mathrm{e} C$ $\left(\mathrm{kg} / \mathrm{m}^{3}\right)$ é a concentração instantânea da solução de acordo com o tempo.

Morse e Arvidson, 2002,estudaram a dissolução de minerais de carbonato na superfície da Terra considerando o mesmo conceito sobre o coeficiente de transferência de massa. Seu modelo consiste na equação,

$$
-\frac{d m_{\text {calcite }}}{d t}=\frac{A}{V} k(1-\Omega)^{n},
$$

onde $m_{\text {calcite }}$ é o número de mols de calcita, $t(\mathrm{~h})$ é o tempo, $A\left(\mathrm{~m}^{2}\right)$ é a área superficial total do sólido, $V\left(\mathrm{~m}^{3}\right)$ é o volume da solução, $k(\mathrm{~m} . \mathrm{mol} / \mathrm{h})$ é $\mathrm{o}$ coeficiente de transferência de massa, $n$ (adimensional) é uma constante positiva que expressa a ordem da reação e $\Omega$ (adimensional) é o estado de saturação.

Finneram e Morse, 2009, apresentaram um estudo da cinética de dissolução de calcita em águas salinas baseado no modelo proposto,

$$
R=k^{*}\left(1-\Omega_{\text {calcite }}\right)^{n},
$$

onde $R\left(\mathrm{~mol} / \mathrm{m}^{2} . \mathrm{h}\right)$ é o fluxo de dissolução normalizada na superfície da reação, $k$ $\left(\mathrm{mol} / \mathrm{m}^{2} . \mathrm{h}\right)$ é o coeficiente de transferência de massa, $\Omega_{\text {calcite (adimensional) é o }}$ estado de saturação e $n$ (adimensional) é a ordem da reação.

Alkattan et al., 1997,estudaram a dissolução cinética da halita $(\mathrm{NaCl})$ considerando o modelo cinético em relação a cada íon,

$$
\frac{d m_{N a^{+}}}{d t}=\frac{d m_{C l^{-}}}{d t}=k_{t}\left(C^{*}-C\right),
$$

onde $k_{t}$ é o coeficientede transferência de massa $\left(\mathrm{m}^{3} / \mathrm{h}\right)$, determinado pela relação entre o coeficiente de difusão e um coeficiente empírico de camada limite, 


$$
k_{t}=\frac{D_{\delta}}{\delta}
$$

Vários trabalhos propõem que o coeficiente de transferência de massa pode ser estimado por meio do número de Sherwood,

$$
S h=\frac{k L}{D_{\delta}}
$$

onde $S h$ é o número de Sherwood, $k$ é o coeficiente de transferência de massa, $L$ é um comprimento característico e $D_{\delta}$ é o coeficiente difusivo de transferência de massa determinado por meio do número de Schmidt,

$$
S c=\frac{\mu}{D_{\delta} \rho},
$$

onde $S c$ é o número de Schmidt, $\mu$ é a viscosidade dinâmica do solvente e $\rho$ é a massa específica do solvente. E por meio do número de Arquimedes,

$$
A r=\frac{g L^{3} \rho\left(\rho_{s}-\rho\right)}{\mu^{2}},
$$

onde $A r$ é o número de Archimedes, $g$ é a aceleração da gravidade e $\rho_{s}$ é a massa específica do sal.

Aksel rud et al.,1992, observaram a partir dos dados experimentais obtidos em seu trabalho, a seguinte relação entre os números adimensionais,

$$
S h=0.31 \sqrt[3]{S c} \sqrt[3]{A r}
$$

Esta correlação é válida para geometria cilíndrica e somente o efeito da temperatura sobre a cinética está sendo considerado.

$\mathrm{Na}$ equação 10 o número de Sherwood é descrito em função do número de Reynolds e Schmidt. Neste caso, o efeito da turbulência é considerado por Bird et al. (2002),

$$
S h=2+0.6 \sqrt[2]{\operatorname{Re} \sqrt[3]{S c}}
$$

onde $R e$ é o número de Reynoldsdefinido como

$$
R e=\frac{d v \rho}{\mu}
$$

se o escoamento for de um fluido newtoniano em um duto cilíndrico, por exemplo. A variável $d$ é um comprimento característico, vé a velocidade média do escoamento, $\rho$ é a massa específica e $\mu$ é a viscosidade dinâmica.

O modelo cinético para a dissolução durante escoamento é representado pela equação

$$
\frac{\partial C}{\partial t}+v_{z} \frac{\partial C}{\partial z}=G
$$

onde $C$ é a concentração do soluto no solvente, $v_{z}$ é a velocidade do fluido na direção $z$ e $G$ é o termo de geração ou consumo do soluto em questão.

\subsection{Tanques de agitação}

Para projetos de tanques de agitação, as correlações encontradas em Nagata (1975) foram utilizadas como referência.

O número de Reynolds que expressa o regime de escoamento dentro do tanque é dado por

$$
R e=\frac{N D^{2} \rho}{\mu},
$$

onde $N$ é o número de rotações por unidade de tempo, geralmente em RPM, RPH ou $\mathrm{Hz}$ e $D$ é o diâmetro do impelidor.

Outro número adimensional relevante para o dimensionamento de um tanque agitado e com sólidos em suspensão é o número de fluxo, definido como,

$$
N_{0}=\frac{Q}{N D^{3}},
$$

onde $Q$ é a vazão volumétrica causada pelo impelidor no tanque.

Um método utilizado pela indústria para definir o número de rotações necessárias para manter sólidos em suspensão em um sistema de tanque agitado é aproximar o impelidor a uma 
bomba centrífuga. A vazão requerida para manter os sólidos em suspensão em função do fator de homogeneidade é (Semco, 2008),

$Q_{r}$

$=618 T_{t}^{2} F v_{t} \beta\left[\left(6.9 \frac{H}{T_{t}}\right)\right.$

$-0,9] \gamma$

em que,

$\beta=(\log \sqrt{f}+0,6)$,

$\gamma=\left(\log \left(T_{t}\right)+1,02\right)$

onde $Q_{r}$ éa vazão requerida, $T_{t}$ é o diâmetro do tanque, $F$ é o fator de homogeneidade, $v_{t}$ é a velocidade terminal da partícula em suspensão, $f$ é a fração mássica de sólidos e $H$ é a altura do fluido dentro do tanque.

$\mathrm{O}$ fator $\mathrm{F}$ é escolhido de acordo com a Tabela 1 (Semco, 2008).

Tabela 1.Fator de homogeneidade.

\begin{tabular}{cl}
\hline Fator & Estado de suspensão dos sólidos \\
$F$ & \\
\hline 1 & Depositados. \\
2 & Depositados em movimento. \\
3 & Parte depositado, parte suspenso. \\
4 & Suspensos, pouca \\
& homogeneidade. \\
5 & Suspensos, média \\
6 & homogeneidade. \\
\hline
\end{tabular}

Se $Q$ é a vazão gerada pelo impelidor dentro do tanque e $Q_{r}$ a vazão requerida para manter o sistema de acordo com a descrição encontrada na tabela 1 , então para estimar aproximadamente o número de rotações necessárias do impelidor admite-se $Q=Q_{r}$. Substituindo a equação 15 em 14 obtém-se,

$N$

$=618 T_{t}^{2} F v_{t} \beta\left[\left(6,9 \frac{H}{T_{t}}\right)\right.$

$-0.9] \gamma \delta$, onde,

$\delta=\left(\frac{1}{N_{0} \cdot D^{3}}\right)$

sendo $N_{0}$ encontrado na Tabela 2 , de acordo com o tipo de impelidor (Semco, 2008).

Tabela 2.Número de fluxo para impelidores.

\begin{tabular}{lc}
\hline \multicolumn{1}{c}{ Tipo de impelidor } & $N_{0}$ \\
\hline Hydrofoil HE & $0,45-$ \\
& 0,65 \\
Hydrofoil LS & $0,50-$ \\
& 0,72 \\
Hydrofoil HS & $0,90-$ \\
& 1,60 \\
Marine Impeller (Helix) & 0,72 \\
\hline
\end{tabular}

Todas as correlações apresentadas são válidas para projeto com dimensões geométricas dentro da variação permitida. Caso contrário, é preciso buscar correlações que contenham referências matemáticas explícitas para a geometria do tanque, impelidor e posição do rotor. Na Tabela 3 é mostrada a faixa de projeto convencional para a geometria do tanque e do rotor(NAGATA 1975).

Tabela 3. Dimensões de projeto para tanques de agitação.

\begin{tabular}{lc}
\hline Razão & $\begin{array}{c}\text { Dimensões para o projeto } \\
\text { (NAGATA, 1975) }\end{array}$ \\
\hline$D / T$ & $1 / 4$ a $1 / 3$ \\
$H / T$ & 1 \\
$C / T$ & $1 / 6$ a $1 / 2$ \\
$B_{W} / T$ & $1 / 12$ a $1 / 10$ \\
\hline D - Diâmetro do impelidor, $T$ - Diâmetro do tanque, $\mathrm{H}-$ Altura \\
do liquido dentro do tanque, $\mathrm{C}-$ - Distância entre o fundo do \\
tanque e o impelidor, $\mathrm{B}_{\mathrm{W}}-$ - largura da chicana.
\end{tabular}

\section{Materiais e Métodos}

\subsection{Caracterizações do sal e do solvente}

O sal adotado para o estudo foi o cloreto de sódio comercial. Cada amostra a ser dissolvida foi considerada como um conjunto de partículas, e foi devidamente caracterizada quanto a sua composição química e faixa granulométrica. A Tabela 4 mostra a composição química típica e a Figura 1 mostra a distribuição de tamanho 
típica da população de partículas, na qual y é a fração mássica não retida e d o diâmetro.

Tabela 4. Composição das amostras.

\begin{tabular}{lc}
\hline \multicolumn{1}{c}{ Substância } & Composição \\
\hline $\mathrm{NaCl}$ & $>99 \%$ \\
$\mathrm{KIO}_{3}$ & $<1 \%$ \\
$\mathrm{Na}_{4} \mathrm{Fe}(\mathrm{CN})_{6}$ & $<1 \%$ \\
\hline
\end{tabular}

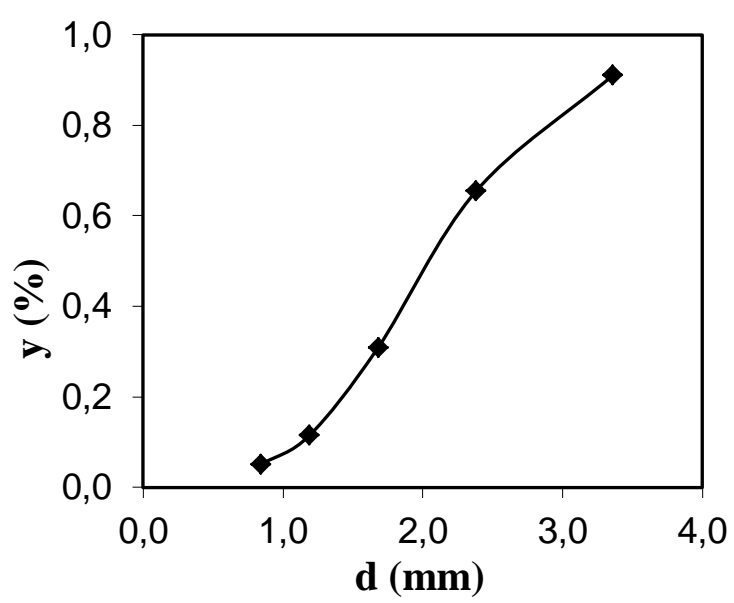

Figura 1. Distribuição de tamanho.

Usualmente utiliza-se água destilada como solvente na determinação da cinética de dissoluções, mas como a mesma não representa a realidade na indústria, este trabalho adotou água potável como meio solvente. É entendido que a presença de um íon comum afeta a cinética de dissolução (Prausnitz, 2000), por tanto, para o escopo deste trabalho, este efeito foi contabilizado de forma implícita ao experimento, embutido no valor dos parâmetros obtidos a partir dos dados experimentais. A composição química típica está apresentada na Tabela 5.

Tabela 5. Composição do solvente (água potável).

\begin{tabular}{lc}
\hline \multicolumn{1}{c}{ Substância } & Composição (mg/l) \\
\hline $\mathrm{SrSO}_{4}$ & 0.02 \\
$\mathrm{CaSO}_{4}$ & 3.40 \\
$\mathrm{MgSO}_{4}$ & 0.99 \\
$\mathrm{Mg}_{4}\left(\mathrm{HCO}_{3}\right)_{2}$ & 1.46 \\
$\mathrm{Mg}\left(\mathrm{NO}_{3}\right)_{2}$ & 0.24 \\
$\mathrm{KNO}_{3}$ & 0.33 \\
$\mathrm{KCl}$ & 1.09 \\
$\mathrm{NaCl}$ & 15.25 \\
$\mathrm{Al}_{2} \mathrm{O}_{3}$ & 0.11 \\
\hline
\end{tabular}

\subsection{Unidade bancada}

A construção desta unidade consistiu em determinar as dimensões geométricas de acordo com a Tabela 3, resultando na compra de um tanque com 8 litros de capacidade, um agitador mecânico de $0,5 \mathrm{cv}$, um termopar e uma resistência para aquecimento. As dimensões finais do sistema construído são: $D / T$ igual a $1 / 3, H / T$ igual a $1, C / T$ igual a $1 / 3$ e $B_{w} / T$ igual a $1 / 10$.

$\mathrm{Na}$ Figura 2 pode ser observado o esquema de montagem desta unidade.



Figura 2. Unidade de mistura em bancada. 1 - tanque de mistura, 2 - trocador de calor, 3 - termopar e 4 agitador mecânico com tacômetro.

$\mathrm{O}$ procedimento experimental consistiu em verter a amostra no centro do tanque, por meio de um funil, onde a água estava a uma temperatura desejada e sob constante agitação. Em tempos aleatórios, amostras da solução eram coletadas do tanque e sua concentração final aferida por meio de técnicas gravimétricas e condutivimetria.

O planejamento experimental foi realizado em análise fatorial. As variáveis independentes são o número de Reynolds e a temperatura. A variável dependente é a concentração final da solução. A concentração inicial foi sempre zero e o volume de solvente foi sempre o mesmo para todos os experimentos. 


\subsection{Modelagem proposta para a dissolução do cloreto de sódio na unidade experimental de bancada \\ $\mathrm{O}$ modelo proposto para a} dissolução no tanque de mistura é baseado no acúmulo de sal na fase líquida, sendo assim, com base na Equação 1 foi proposta a mudança,

$$
\frac{d C}{d t}=\frac{k A\left(C^{*}-C\right)}{V},
$$

onde $C\left(\mathrm{~kg} / \mathrm{m}^{3}\right)$ é a concentração instantânea do sal na solução, $t(\mathrm{~s})$ é o tempo, $k(\mathrm{~m} / \mathrm{s})$ é o coeficiente de transferência de massa, $A\left(\mathrm{~m}^{2}\right)$ é a área superficial da amostra, $C^{*}\left(\mathrm{~kg} / \mathrm{m}^{3}\right)$ éa concentração de saturação e $V\left(\mathrm{~m}^{3}\right)$ é o volume da solução.

Para definir a área superficial, inicialmente considerou-se a partícula como uma esfera perfeita, a área média é descrita por,

$$
A_{p}=4 \pi\left(\overline{\frac{D_{p}}{2}}\right)^{2}
$$

onde $A_{p}$ é a área superficial da partícula $\mathrm{e}$ $\overline{D_{p}}$ é o diâmetro médio de Sauter.

O diâmetro médio de Sauter é definido pela equação (Brennen, 2005),

$$
\overline{D_{p}}=\frac{1}{\int_{0}^{1} \frac{d X}{D_{p}}} \cong \frac{1}{\sum_{i} \frac{\Delta X_{i}}{D_{p i}}}
$$

onde $\Delta X_{i}$ é a massa retida na malha e $D_{p i}$ é o diâmetro da malha.

Para o volume médio de uma esfera tem-se,

$$
V_{p}=\frac{4}{3} \pi\left(\overline{\frac{D_{p}}{2}}\right)^{3}
$$

onde $V_{p}$ é o volume da partícula.

Para a massa específica tem-se,

$$
\rho_{s}=\frac{m_{p}}{V_{p}}
$$

onde $\rho_{s}$ é a massa específica do sal e $m_{p}$ é a massa da partícula.

Combinando as equações 18, 20 e 21 pode-se obter uma equação para a área média superficial de uma partícula em função da massa,

$$
A_{p}=\pi\left(\frac{6 m_{p}}{\rho_{s} \pi}\right)^{\frac{2}{3}} .
$$

Para a área superficial de um conjunto de partículas, foi proposta uma generalização da equação 22. Onde, ao invés de usar a massa da partícula, usa-se a massa total da população dividida pelo número de partículas. O resultado é a massa média de uma partícula que pertence à população. Multiplicando isso pelo número de partículas, o resultado é uma aproximação da área média superficial total. Introduzindo um fator adimensional que corrige a área para qualquer geometria tem-se,

$$
A=\frac{\pi N p}{\varphi}\left(\frac{6\left(M-V\left(C-C_{i}\right)\right.}{\rho_{s} \pi N p}\right)^{\frac{2}{3}}
$$

onde $A\left(\mathrm{~m}^{2}\right)$ é a área superficial média da população de partículas, $N p$ (adimensional) é o número de partículas, $C\left(\mathrm{~kg} / \mathrm{m}^{3}\right)$ é a concentração instantânea em função do tempo, $C_{i}$ $\left(\mathrm{kg} / \mathrm{m}^{3}\right)$ é a concentração inicial da solução, $\varphi$ (adimensional) é a esfericidadee $M(\mathrm{~kg})$ é a massa da população.

Segundo Massarani, 2000, processos de moagem e quebra geram partículas com esfericidade entre $0,7 \mathrm{e}$ 0,8 . Este trabalho utilizou 0,7 nos cálculos.

Para o número de partículas, utilizou-se o volume total da população dividida pelo volume médio de uma partícula. Depois de manipulação algébrica:

$$
N p=\frac{M}{\frac{4}{3} \rho_{s} \pi\left(\frac{\overline{D_{p}}}{2}\right)^{3}} .
$$




\subsection{Unidade experimental de escoamento.}

Esta unidade foi composta basicamente por três calhas com6metros de comprimento, estrutura para sustentação, tanque de abastecimento e receptor, alimentador de sólidos (Laboreto), válvulas, uma bomba centrífuga e comandos elétricos. $\mathrm{O}$ procedimento experimental consistiu em preparar a salmoura no tanque de alimentação, calibrar a vazão volumétrica da mesma nas calhas, calibrar a vazão mássica do alimentador de sólidos e determinar também sua posição nas calhas. Em seguida era feito o bombeio da salmoura para as calhas, onde também a amostra de sal era alimentada. Como consequência, ambos escoavam juntos até o final da linha onde eram coletados, separados, e o sal que não foi solubilizado submetido à secagem convectiva. Como principais dados de saída do experimento, tinha-se a massa total de sal seco e a concentração final da solução. $\mathrm{O}$ esquema pode ser visto na Figura 3.

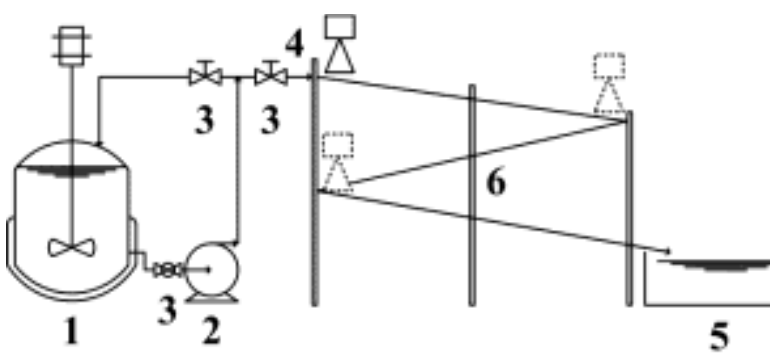

Figura 3.Desenho esquemático da unidade experimental de escoamento de sais. 1 - tanque de mistura, 2 - bomba centrífuga, 3 - válvulas, 4 alimentador de sólidos, 5 - tanque receptor e 6 - sistema de calhas.

A malha experimental foi baseada em uma análise fatorial tomando como referência dois níveis e quatro fatores resultando em dezesseis experimentos. A variável dependente é a concentração final da salmoura e as independentes são vazão volumétrica, vazão mássica, comprimento de residência do sal e concentração inicial da salmoura.

\subsection{Modelagem proposta para a} dissolução do cloreto de

\section{sódio \\ na \\ unidade experimental de escoamento}

Neste modelamento, o processo dissolutivo ocorre na superfície do sal (Prausnitz, 2000),e para a simplificação dos cálculos, foi considerada a aproximação de que a interface sólidolíquido e solução-ambiente estão em equilíbrio térmico. Também foi admitida a hipótese experimental de que a velocidade relativa entre o sólido e a solução é zero.

$\mathrm{O}$ balanço de massa para o soluto foi considerado tanto na fase líquida quanto na sólida, resultando num sistema de duas equações diferenciais parciais.

A equação 25 representa o balanço de massa do sal na solução e a equação 26 o balanço de massa do sal na fase sólida,

$\left(\frac{\partial}{\partial t} C(z, t)\right)+\bar{v}\left(\frac{\partial}{\partial z} C(z, t)\right)$
$=k a\left(C^{*}-C(z, t)\right)$,

$\rho_{s}\left(\frac{\partial}{\partial t} \varepsilon_{s}(z, t)+\bar{v}\left(\frac{\partial}{\partial z} \varepsilon_{s}(z, t)\right)\right)$

$=-k a\left(C^{*}-C(z, t)\right)$,

onde $C\left(\mathrm{~kg} / \mathrm{m}^{3}\right)$ é a concentração da solução de acordo com o tempo e posição, $t(\mathrm{~s})$ é o tempo, $z(\mathrm{~m})$ é a posição, $\bar{v}(\mathrm{~m} / \mathrm{s})$ é a velocidade média da solução durante o escoamento, $k(\mathrm{~m} / \mathrm{s})$ é o coeficiente de transferência de massa, $a\left(\mathrm{~m}^{-1}\right)$ é a área específica de transferência de massa, $C^{*}$ $\left(\mathrm{kg} / \mathrm{m}^{3}\right)$ é a concentração de saturação da solução, $\rho_{s}\left(\mathrm{~kg} / \mathrm{m}^{3}\right)$ é a massa específica do sal e $\varepsilon_{s}$ (adimensional) é a fração volumétrica de sólidos.

A área específica é definida pela razão entre a área superficial média total de transferência de massa e o volume de controle,

$$
a=\frac{6 \varepsilon_{s}(z, t)}{\overline{D_{p}}}
$$

\subsection{Correlação proposta para a determinação do coeficiente de transferência de massa}


Com os modelos definidos para ambas as unidades experimentais, é necessário informar que correlação será usada para representar a cinética de dissolução, apresentada neste trabalho pela variável $k$. Como o sistema experimental construído é singular, foram propostas novas correlações para representá-lo, em que,

$$
S h=A 0\left(R e^{*}\right)^{A 1}(S c)^{\frac{1}{3}},
$$

onde,

$$
A 0=(P 1+P 2(T-318)),
$$

e

$$
A 1=P 3,
$$

desta forma,

$$
k=A 0\left(\frac{D_{\delta}}{L}\right)\left(R e^{*}\right)^{A 1}(S c)^{\frac{1}{3}} .
$$

Assim, $P 1, P 2$ e $P 3$ são parâmetros experimentais, $R e^{*}$ é uma modificação do número de Reynolds, $\frac{D_{\delta}}{L}$ é o coeficiente de difusão característico e Scé o número de Schmidt.

O coeficiente de difusão para o cloreto de sódio em água a $298 \mathrm{~K}$ é $1.61 .10^{-9} \mathrm{~m}^{2} / \mathrm{s}$ (Cremasco, 2000). Para o efeito da temperatura,

$$
D_{\delta}=\frac{\alpha T}{\mu},
$$

sendo $\alpha$ uma constante estimada à uma temperatura de referência, $D_{\delta}$ é o coeficiente difusivo, $\mu$ é a viscosidade dinâmica do solvente eTa temperatura do solvente.

Para o comprimento característico do sistema, $L$, adotou-se $\mathrm{o}$ diâmetro externo da partícula de sal, portanto este valor vem da análise granulométrica da amostra.

Para expressar o grau de turbulência do sistema, propôs-se um número de Reynolds modificado, que não fosse função da geometria de escoamento. Matematicamente é um número normalizado, que varia de 0 a 1 , representado pela equação,

$$
R e^{*}=\frac{R e-R e_{c}}{R e},
$$

onde $R e_{c}$ é o número de Reynolds crítico.

O número de Reynolds crítico é o grau mínimo de turbulência necessário para manter uma distribuição homogênea das partículas de sal no sistema. Todos os modelos propostos até aqui são válidos apenas para este tipo de sistema.

\section{Resultados e Discussão}

\subsection{Resultados experimentais obtidos para a unidade de bancada}

$\mathrm{Na}$ Tabela em anexo são apresentados os resultados obtidos para o coeficiente de transferência de massa, por meio da substituição dos dados experimentais de concentração e tempo na equação 17. Cada experimento foi feito em triplicata. Pelo fato do modelo proposto apresentar uma correlação explícita para a correção da área de troca mássica a cada mudança de concentração da solução, o parâmetro $k$, a cada instante, pôde ser obtido pela solução analítica da equação 17. A integração da mesma e o isolamento de $k$ levam à seguinte equação,

$k=\left(-\ln \left[\frac{C^{*}-C}{C^{*}-C_{i}}\right]\right) \cdot\left(\frac{V}{t . A}\right)$

ressaltando que $C$ é a concentração instantânea, neste caso, a concentração experimental, e $A$ é calculado a cada ponto experimental de concentração, segundo a equação 23 proposta.

Observa-se na Tabela em anexo que houve um aumento na cinética de dissolução neste sistema à medida que a temperatura e a velocidade de rotação aumentam. O que é esperado, pois são duas condições operacionais que influenciam diretamente na camada limite de transferência de massa existente entorno da partícula de sal. Também pode ser observado que existem proporções a serem resguardadas de experimento para 
experimento, por exemplo: o aumento da cinética causado na diferença existente entre os experimentos 1 e 3 não é o mesmo causado entre o 7 e 9.

Os resultados contidos na Tabela em anexo foram utilizados para a obtenção dos parâmetros experimentais P1, P2 e P3. Com seus valores definidos, esta equação passa a representar a cinética da dissolução em bancada, e será utilizada para a resolução do sistema representado pelas equações 25 e 26.

Para encontrar o valor destes parâmetros foi feito a substituição da equação 29 na equação 17 , resultando numa estimação de parâmetros em uma equação diferencial.

Para a solução, foi desenvolvido um algoritmo matemático em linguagem MAPLE que busca por meio de séries de potência o polinômio que melhor representa a solução literal da equação diferencial, e estima, a partir deste polinômio, os parâmetros experimentais de acordo com a alimentação dos dados experimentais (variáveis dependentes e independentes).O polinômio ajustado está demonstrado na equação 33 ,

C

$=7,0 \cdot 10^{5} \cdot k \cdot A \cdot t-7,8 \cdot 10^{6} \cdot k^{2} \cdot A^{2} \cdot t^{2}$

$+5.7 \cdot 10^{8} \cdot k^{3} A^{3} t^{3}$

onde $k$ e $A$ são substituídos pelas equações 29 e 23 e t é o tempo. As demais substituições dentro das equações de $k$ e $A$ estão anteriormente detalhadas.

Na Tabela 6 podem ser observados os parâmetros médios obtidos, com tolerância de $10^{-5}$ para a parada de iterações da função de minimização do erro.

Tabela 6.Parâmetros obtidos

\begin{tabular}{cc}
\hline $\begin{array}{c}\text { Parâmetro } \\
\text { (adimensional) }\end{array}$ & Valor \\
\hline P1 & 7,40 \\
P2 & 0,19 \\
P3 & 0,06 \\
\hline
\end{tabular}

\subsection{Resultados experimentais obtidos para a unidade de escoamento}

Na Tabela 7 são mostrados os dados experimentais obtidos na unidade de escoamento. Nesta tabela observam-se todas as variáveis independentes do processo, que são as próprias condições operacionais no qual o sistema funcionou, bem como a variável final de interesse, denominado FD. Esta é a variável dependente que expressa a quantidade de massa de sal dissolvida, calculada pela equação 34 ,

$$
F D=\left(\frac{m_{i}-m_{f}}{m_{i}}\right) \cdot 100
$$

onde $m_{i}$ é a massa inicial para dissolução (massa da amostra no Laboreto) e $m_{f}$ é a massa restante de sal, coletada ao final das calhas de escoamento. A massam é aferida após um processo de secagem.

Este resultado implicitamente fornece a concentração final da salmoura.

As variáveis independentes foram descritas da seguinte maneira: $C$ é concentração inicial da salmoura previamente preparada no tanque de alimentação, W é a vazão mássica de sal alimentada na calha, CR é o comprimento de residência e Q é a vazão volumétrica da salmoura.

Tabela 7.Resultados experimentais para unidade de escoamento.

\begin{tabular}{c|c|c|c|c|c}
\hline Exp. & $\mathrm{C}(\mathrm{g} / \mathrm{l})$ & $\mathrm{W}(\mathrm{g} / \mathrm{s})$ & $\mathrm{CR}(\mathrm{m})$ & $\mathrm{Q}(1 / \mathrm{s})$ & $\mathrm{FD}(\%)$ \\
\hline 1 & 94,0 & 11,01 & 18 & 0,53 & 74,15 \\
2 & 94,0 & 24,05 & 18 & 0,54 & 72,77 \\
3 & 94,0 & 12,36 & 6 & 0,50 & 49,54 \\
4 & 94,0 & 24,51 & 6 & 0,53 & 54,07 \\
5 & 92,3 & 12,52 & 6 & 0,99 & 45,85 \\
6 & 92,3 & 26,19 & 18 & 0,99 & 70,63 \\
7 & 93,3 & 11,35 & 18 & 0,94 & 67,82 \\
8 & 93,3 & 23,69 & 6 & 0,96 & 38,80 \\
9 & 182,3 & 11,62 & 18 & 0,55 & 55,45 \\
10 & 182,3 & 26,05 & 18 & 0,57 & 55,51 \\
11 & 182,3 & 25,11 & 6 & 0,56 & 32,78 \\
12 & 182,3 & 11,81 & 6 & 0,57 & 28,00 \\
13 & 183,0 & 11,19 & 6 & 0,95 & 26,47 \\
14 & 183,0 & 25,66 & 18 & 0,96 & 54,42 \\
15 & 178,3 & 12,14 & 18 & 0,94 & 47,44 \\
16 & 178,3 & 24,09 & 6 & 0,94 & 24,33 \\
\hline
\end{tabular}

Comparando-se o experimento 1 com o 9, percebe-se que a cinética de dissolução é mais lenta quanto maior for a concentração inicial da salmoura, salvo as devidas proporções operacionais 
existentes entre os respectivos experimentos. Isto é esperado já que a força motriz de transferência de massa é baseada no gradiente de concentração existente entre o sal sólido e a fase líquida. A mesma tendência é observada, por exemplo, quanto se compara o experimento 2 e 10 .

Isto se demonstra matematicamente por meio das equações 25 e 26. A taxa de geração de massa no meio será nula se $C^{*}=C$.

$\mathrm{O}$ mesmo raciocínio pode ser estendido às outras variáveis operacionais. Para a vazão mássica de sólidos $W$, as tendências são contraditórias. Neste ponto, entende-se que o efeito da concentração de sólidos não é relevante frente aos outros efeitos que ocorrem simultaneamente, ou somente para este sistema, é desprezível devido às proporções geométricas e operacionais. Para a variável CR, percebe-se que quanto maior o comprimento de calha a ser percorrido maior é a dissolução. Este efeito não está relacionado com a cinética diretamente, e sim com o tempo de residência do sal em contato com o fluido. É esperado que quanto maior o tempo de residência maior a massa dissolvida. A vazão volumétrica relaciona-se também com o tempo de residência, já que maiores vazões levam a maiores velocidades de escoamento.

Para a determinação da cinética no sistema de escoamento, foi utilizada a correlação 28 com os parâmetros obtidos para a cinética na unidade em bancada (Tabela 6).

Desta forma, o estudo da cinética de dissolução na unidade de escoamento se deu de forma indireta, por meio da comparação entre a concentração experimental final da salmoura e a calculada pelo modelo composto pelas equações 25,26 e 29.

Os desvios entre estas concentrações apontarão para dois caminhos, primeiro: quanto maior o desvio, menos semelhança há entre as cinéticas existentes no tanque e calha. Segundo: caso os desvios sejam baixos, isto indicará que para os sistemas adotados em suas respectivas dimensões e condições operacionais, o comportamento da camada limite foi semelhante. Esta metodologia pode representar uma vantagem sob o ponto de vista matemático: obter-se uma cinética num sistema mais simples para extrapolá-la num sistema mais complexo.

\subsection{Resultados obtidos para a utilização da cinética de bancada no modelo da unidade de escoamento}

O sistema a ser resolvido proveniente da modelagem da unidade experimental de escoamento ficou definido pelas equações 25 e 26 , tomando como cinética o modelo obtido a partir dos experimentos em bancada, descrito pela equação 29 .

Este sistema é um sistema diferencial parcial implícito. Baseado no método das diferenças finitas centrais esse sistema foi resolvido novamente em linguagem MAPLE.

Na Figura 4 observa-se o resultado obtido para a comparação entre a concentração final da salmoura e a concentração calculada pelo modelo em questão, na mesma posição de referência.

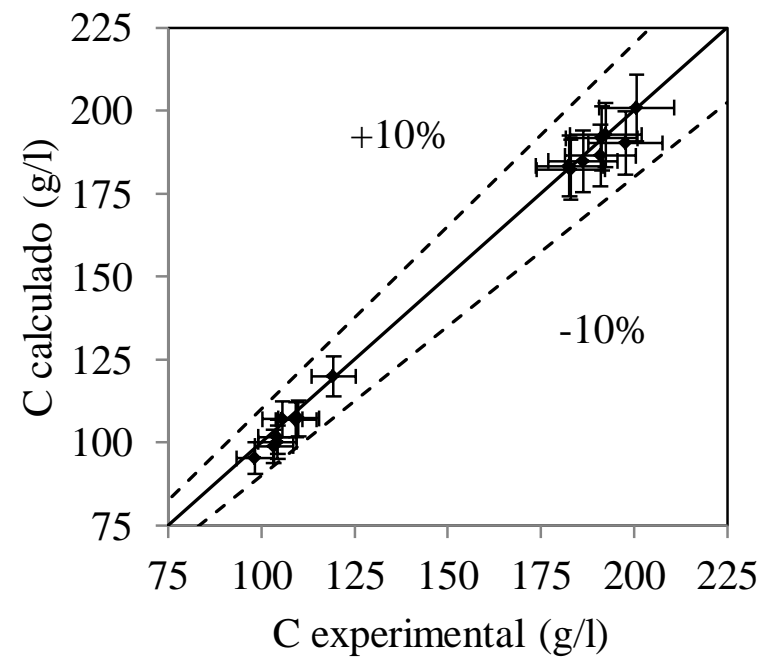

Figura 4.Concentração experimental versus calculada para unidade de escoamento.

Observa-se na figura que a comparação entre o valor experimental e o calculado utilizando a cinética obtida na bancada produz desvios inferiores a $10 \%$. Foi também analisado a propagação dos 
erros experimentais que não ultrapassaram $5 \%$, representado no gráfico pelas barras verticais e horizontais. Os resultados demonstrados indicam que para este caso em particular, a cinética presente tanto no tanque de mistura quanto na unidade de escoamento são semelhantes, o que leva a especulação de que o coeficiente de transferência de massa seja similar em termos de valor numérico.

A literatura afirma que a transferência de massa é controlada pela formação da camada limite de troca mássica (Bird et al., 2002), sendo assim, é possível que o comportamento da camada limite em ambos os processos dissolutivos foi semelhante, o que justificaria os resultados contidos na figura 4. Outra hipótese é que para ambos os sistemas o tempo de residência do sal é pequeno, e similar para cada experimento nas duas unidades. Isto poderia causar uma amortização nos efeitos de temperatura e turbulência que possam estar presentes em uma configuração dissolutiva e não na outra.

\section{Conclusões}

Os resultados demonstraram que, para este sistema em particular, a cinética de dissolução presente tanto na bancada quanto na unidade de escoamento são semelhantes. Isto significa entender que o valor dos coeficientes de transferência de massa para ambas as unidades são similares. Ainda não se pode afirmar se esta é uma metodologia válida para qualquer dimensão de sistema. Em trabalhos futuros novas condições de estudo serão criadas para verificar a extensão da dependência entre o processo dissolutivo e a geometria do sistema.

\section{Referências}

AKSEL'RUD, G.A, BOIKO, A.E, KASHCHEEV, A.E. "Kinectis of the solution of mineral salts suspended in a liquid flow".UDC 532.73-3 (1992). SPE (Society of Petroleum Engineers).

ALKATTAN M., OELKERS E.H., DANDURAND J.L., SCHOTT J., Elsevier. "Experimental studies of halite dissolution kinetics. The effect of saturation state and the presence of trace metals". Chemical Geology, 137 (1997) 201-219.

BIRD, R.B., STEWART, W.E. \& LIGHTFOOT, E.N. Transport Phenomena.Second Edition.Chemical Engineering Department.University of Wisconsin-Madison, 2002.

BRENNEN, C. E., "Fundamentals of Multiphase flow". Cambridge University Press, 2005.

FINNERAN D.W., MORSE J.W., "Calcite dissolution kinetics in saline waters".Elsevier, chemical geology 268 (2009) 137-146.

MORSE J.W., ARVIDSON R. S., "The dissolution kinetics of major sedimentary carbonate minerals". Elsevier, Earthscience reviews 58 (2002) 51-84.

NAGATA S., "Mixing - Principles and Applications". Kodansha scientific books, 1975.

PRAUSNITZ, J.M, LICHTENTHALER, R.N, AZEVEDO, E.G, "Termodinámica Molecular de los Equilibrios de Fase", Third Edition, Prentice Hall Iberia, Madrid, 2000.

SEMCO PROCESSOS, Palestra "Agitação \& Sólidos em Suspensão? MaiorRendimento X MenorConsumo de Energia", material técnico elaborado em 2008, disponível no link:http://www.semcoequipamentos.com. br/en/misturas/atuacao/aplicacoes/semco_ palestra_solidossuspensao.pdf. Acessado em 21/08/2010.

SHAUGHNESSY, J., DAUGHERTY, W., GRAFF, R., DURKEE, T., 2007.More Ultra-Deepwater Drilling Problems. SPE/IADC Drilling Conference, ID: 105792-MS. ISBN 9781-55563-158- 


\section{Simbologia}

Lista de símbolos organizados por ordem alfabética.

Sigla

Descrição

Unidade

a Área específica de transferência de massa

$m^{-1}$

A Área superficial média da população de partículas

$m^{2}$

$A_{P} \quad$ Área superficial da partícula

$m^{2}$

Ar Número de Archimedes

adimensional

$B_{W} \quad$ Largura da chicana

$m$

C Concentração instantânea

$\mathrm{kg} / \mathrm{m}^{3}$

$C^{*} \quad$ Concentração no ponto de saturação

$\mathrm{kg} / \mathrm{m}^{3}$

$C_{i} \quad$ Concentração inicial da solução

$\mathrm{kg} / \mathrm{m}^{3}$

CR Comprimento de residência

$m$

d Diâmetro característico

$m$

D Diâmetro do impelidor

$m$

$D_{p} \quad$ Diâmetro médio de Sauter

$m$

$D_{p i} \quad$ Diâmetro da malha

$m$

$D_{\delta} \quad$ Coeficiente difusivo de transferência de massa

$\mathrm{m}^{2} / \mathrm{s}$

$D_{\delta / L} \quad$ Coeficiente de difusão característico

$\mathrm{m} / \mathrm{s}$

F $\quad$ Fator de homogeneidade

adimensional

f $\quad$ Fração mássica de sólidos

adimensional

FD Fator de Dissolução

$g \quad$ Aceleração da gravidade

adimensional

G Termo de geração ou consumo do soluto em questão

H Altura do fluido dentro do tanque

$\mathrm{m} / \mathrm{s}^{2}$

$\mathrm{kg} / \mathrm{m}^{3} \cdot \mathrm{s}$

$m$

$k \quad$ Coeficiente de transferência de massa deste trabalho

$m / s$

$k_{t} \quad$ Coeficiente de transferência de massa segundo Alkattan et. al

$m^{3} / h$

L Comprimento característico

$m$

M Massa da população de partículas

$k g$

$m_{f} \quad$ Massa final da amostra de partículas

$k g$

$m_{i} \quad$ Massa inicial da amostra de partículas

$k g$

$m_{p} \quad$ Massa de uma partícula

$k g$

n Constante positiva que expressa a ordem da reação

adimensional

$N \quad$ Número de rotações

$R P M$

$N_{o} \quad$ Número de fluxo

adimensional

$N_{p} \quad$ Número de partículas

adimensional 
P1, P2 e P3 Parâmetros Experimentais

$Q \quad$ Vazão volumétrica

$Q_{r} \quad$ Vazão requerida

$R \quad$ Fluxo de dissolução normalizada na superfície da reação

Re Número de Reynolds

$\operatorname{Re}^{*} \quad$ Número de Reynolds modificado

$R_{c} \quad$ Número de Reynolds crítico

Sc Número de Schmidt

Sh Número de Sherwood

T Temperatura do solvente

$t \quad$ Tempo

$T_{t} \quad$ Diâmetro do tanque

$v \quad$ Velocidade média do escoamento

V Volume da solução

$V_{P} \quad$ Volume de uma partícula

$v_{t} \quad$ Velocidade terminal da partícula em suspensão

W Vazão mássica de sal alimentada na calha

z. Posição

$\alpha \quad$ Constante estimada a uma temperatura de referência

$\delta \quad$ Coeficiente empírico

$\Delta X_{i} \quad$ Massa retida na malha

$\varepsilon_{s} \quad$ Fração volumétrica de sólidos

$\mu \quad$ Viscosidade dinâmica do solvente

$\rho \quad$ Massa específica do solvente

$\rho_{s} \quad$ Massa específica do sal

$\phi \quad$ Esfericidade

$\Omega \quad$ Estado de saturação adimensional

$\mathrm{m}^{3} / \mathrm{s}$
$\mathrm{m}^{3} / \mathrm{s}$
$\mathrm{mol} / \mathrm{m}^{2} . h$
adimensional
adimensional
adimensional
adimensional
adimensional
${ }^{0} \mathrm{C}$
$\mathrm{s}$
$\mathrm{m}$
$\mathrm{m} / \mathrm{s}$
$\mathrm{m}^{3}$
$\mathrm{~m}^{3}$
$\mathrm{~m} / \mathrm{s}$
$\mathrm{kg} / \mathrm{s}$
$\mathrm{m}$

adimensional

$m^{-1}$

$k g$

adimensional

$\mathrm{kg} / \mathrm{m} . \mathrm{s}$

$\mathrm{kg} / \mathrm{m}^{3}$

$\mathrm{kg} / \mathrm{m}^{3}$

Adimensional

Adimensional

\section{Anexo}


Resultados experimentais para a unidade em bancada.

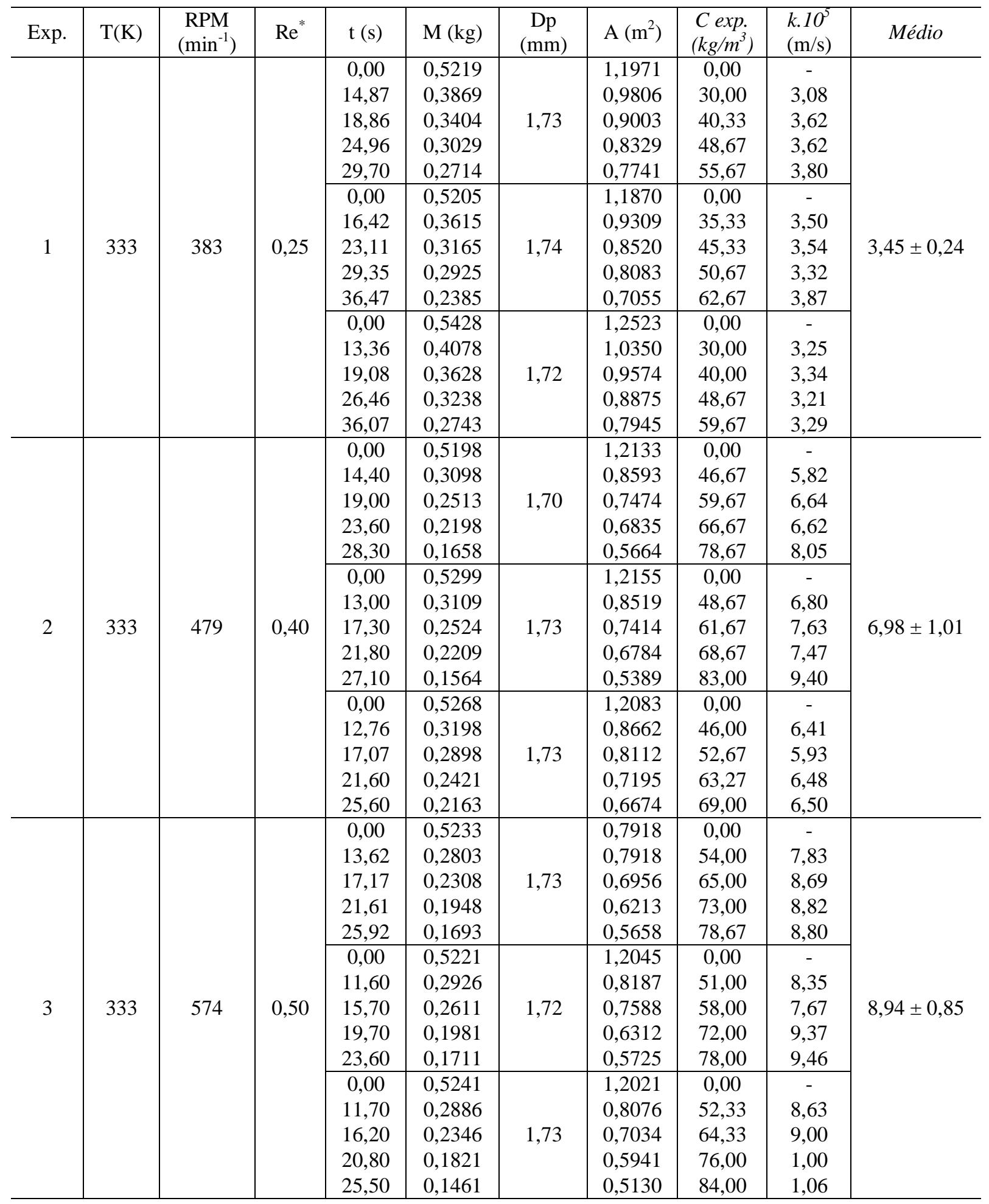




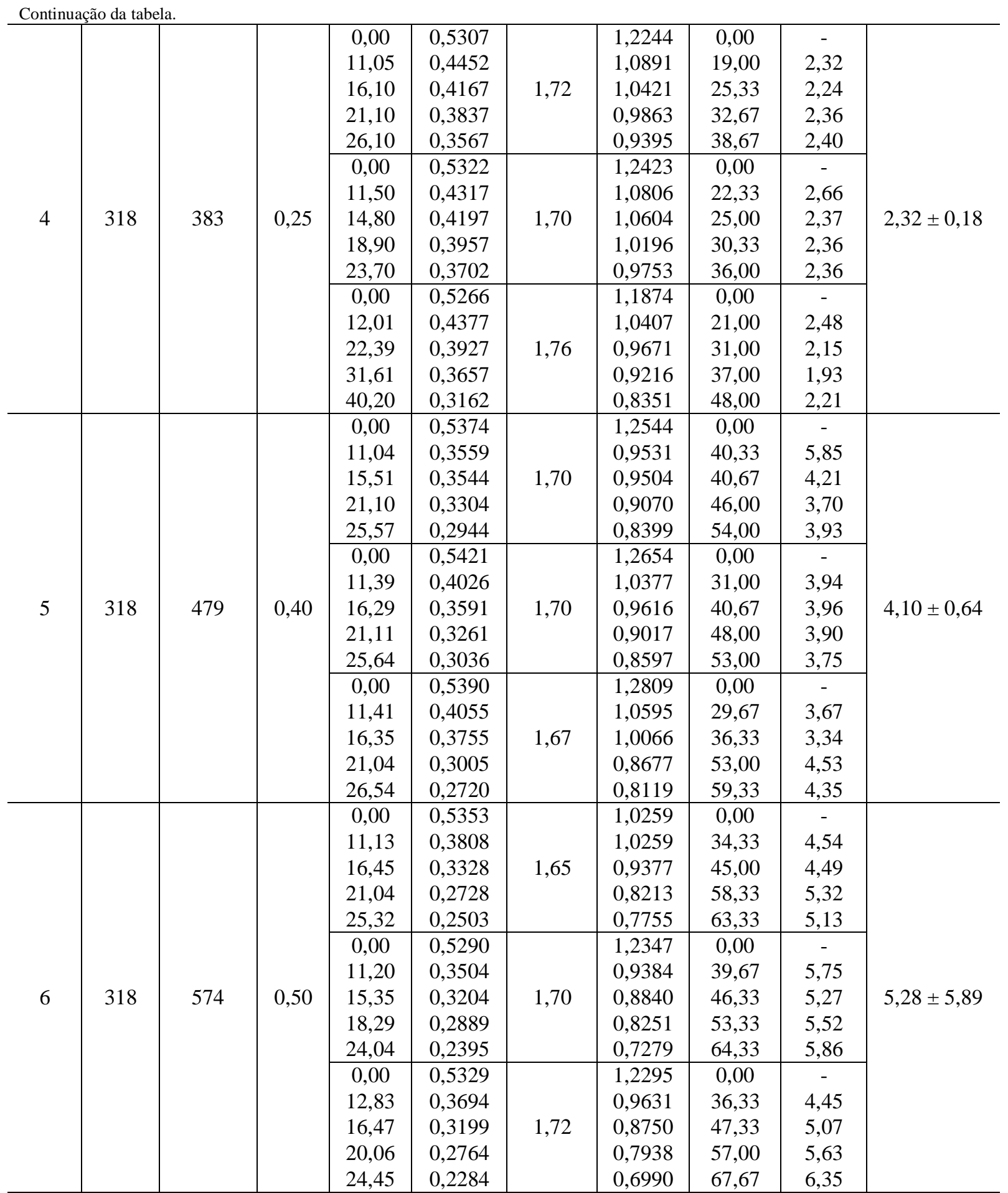




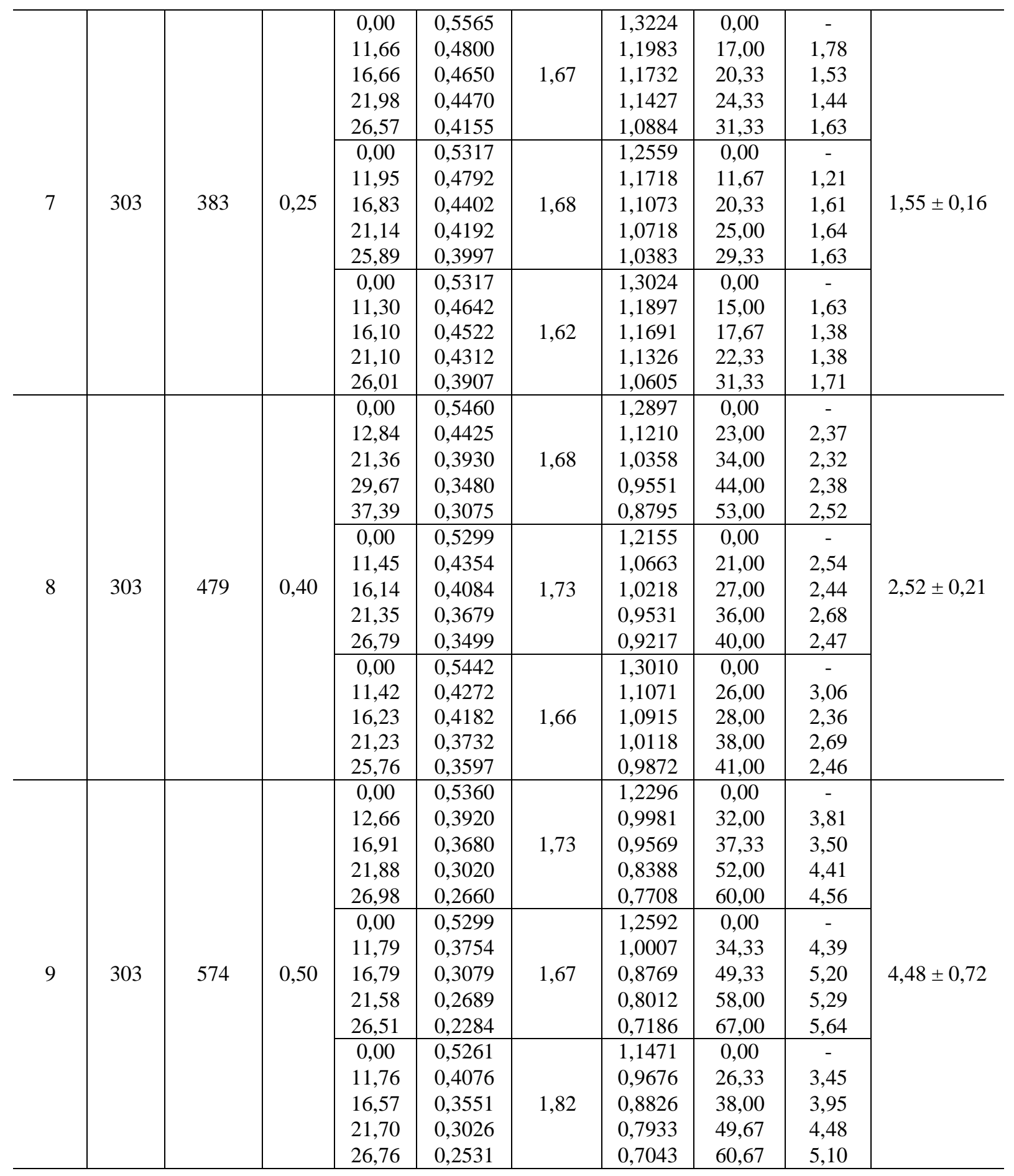

